Proceeding Series of the Brazilian Society of Computational and Applied Mathematics

\title{
Investigando a Influência de Indivíduos Imunes na Propagação de Doenças Contagiosas
}

\author{
Ana Leda Silva Moraes ${ }^{1}$ \\ Programa de Pós-graduação em Engenharia Elétrica e Computação da Universidade \\ Presbiteriana Mackenzie, São Paulo, SP \\ Luiz Henrique Alves Monteiro ${ }^{2}$ \\ Escola de Engenharia da Universidade Presbiteriana Mackenzie e Escola Politécnica da \\ Universidade de São Paulo, São Paulo, SP
}

\begin{abstract}
Resumo. Aqui, propõe-se e analisa-se um modelo epidemiológico, formulado em termos de equações diferenciais ordinárias, que leva em conta o efeito dos recuperados (considerados imunes) na disseminação de infecções que se propagam via contatos sociais entre suscetíveis e infectados. Mostra-se que podem coexistir diferentes soluções estacionárias atratoras.
\end{abstract}

Palavras-chave. Bifurcação, dinâmica populacional, epidemiologia, estabilidade, sistemas dinâmicos.

\section{Introdução}

A propagação de doenças contagiosas vem sendo estudada teoricamente desde 1766, ano do trabalho seminal de Daniel Bernoulli sobre a disseminação da varíola [2]. Um outro trabalho relevante em epidemiologia teórica foi realizado por William O. Kermack e Anderson G. McKendrick, em 1927, sobre epidemias de peste bubônica [4]. Nesses e em outros estudos, costuma-se dividir a população hospedeira em algumas classes e o modelo consiste de equações diferenciais que descrevem a evolução temporal da quantidade de indivíduos em cada classe. Classes típicas em tais estudos são [1,6]: a dos indivíduos suscetíveis $S$ (que são aqueles que podem contrair o agente patogênico por meio de contato com infectados), a dos indivíduos expostos $E$ (que são aqueles que contraíram o patógeno em questão, mas ainda não desenvolveram a doença e não podem propagá-la), a dos indivíduos infectados $I$ (que são aqueles que estão doentes e podem contaminar os suscetíveis via contato social), a dos indivíduos recuperados $R$ (que são aqueles que se curaram, de modo que a cura pode ou conferir imunidade parcial, ou

$171412621 @$ mackenzista.com.br, analeda@uninove.br

2 luizm@mackenzie.br, luizm@usp.br 
total, ou não conferir imunidade), a dos indivíduos mortos $M$ (que são aqueles que morreram ou devido à doença estudada ou por outros motivos), e a dos indivíduos vacinados $V$ (que são aqueles que, ao tomarem uma vacina, adquiriram imunidade ou parcial ou total, sem adoecer).

Em geral, desconsidera-se o efeito da população $R$ sobre a transmissão de uma doença contagiosa [1,6]. Usualmente, tal transmissão é modelada como sendo dependente apenas das populações $S$ e $I$. No estudo aqui relatado, leva-se em conta a influência da população $R$ na propagação de doenças contagiosas. A inspiração para essa investigação vem do seguinte cenário: considere doenças contagiosas típicas de crianças, como catapora, caxumba, sarampo. Em geral, as crianças vivem em seus lares, com seus pais e irmãos, e só saem de casa acompanhadas de seus pais ou de parentes maiores de idade, que as levam para a escola, para o parque, para o shopping. Nesses ambientes, elas entram em contato com outras crianças e lá podem contaminar e serem contaminadas. Note que, nesse cenário, a propagação da doença é facilitada pelos adultos, normalmente imunes a tais doenças. De fato, adultos imunes podem colaborar para o encontro entre crianças sãs e crianças doentes. Assim, a população $R$ pode afetar a taxa de encontro entre as populações $S$ e $I$, interferindo na dinâmica da propagação de uma doença infectocontagiosa.

Este trabalho sobre epidemiologia teórica está assim organizado. Na Seção 2, propõe-se um conjunto de equações diferenciais para realizar o estudo mencionado no parágrafo anterior. Na Seção 3, apresentam-se resultados analíticos. Na Seção 4, mostram-se simulações numéricas, a fim de ilustrar os resultados analíticos relatados na Seção 3. Finalmente, na Seção 5, discute-se a possível relevância deste trabalho.

\section{O modelo proposto}

Considere que $\mathrm{S}(\mathrm{t}), \mathrm{I}(\mathrm{t})$ e $\mathrm{R}(\mathrm{t})$ sejam as quantidades de indivíduos suscetíveis, infectados e recuperados (e imunes), respectivamente, no instante $t$. Em termos de equações diferenciais ordinárias, o modelo proposto se escreve assim:

$$
\begin{aligned}
& \mathrm{dS}(\mathrm{t}) / \mathrm{dt}=\mathrm{f}_{1}(\mathrm{~S}(\mathrm{t}), \mathrm{I}(\mathrm{t}), \mathrm{R}(\mathrm{t}))=-\mathrm{aS}(\mathrm{t}) \mathrm{I}(\mathrm{t})(1+\mathrm{qR}(\mathrm{t}))+\mathrm{cI}(\mathrm{t})+\mathrm{eR}(\mathrm{t}) \\
& \mathrm{dI}(\mathrm{t}) / \mathrm{dt}=\mathrm{f}_{2}(\mathrm{~S}(\mathrm{t}), \mathrm{I}(\mathrm{t}), \mathrm{R}(\mathrm{t}))=\mathrm{aS}(\mathrm{t}) \mathrm{I}(\mathrm{t})(1+\mathrm{qR}(\mathrm{t}))-\mathrm{bI}(\mathrm{t})-\mathrm{cI}(\mathrm{t}) \\
& \mathrm{dR}(\mathrm{t}) / \mathrm{dt}=\mathrm{f}_{3}(\mathrm{~S}(\mathrm{t}), \mathrm{I}(\mathrm{t}), \mathrm{R}(\mathrm{t}))=\mathrm{bI}(\mathrm{t})-\mathrm{eR}(\mathrm{t})
\end{aligned}
$$

A constante de taxa de infecção é a $\underline{a}$, a constante de taxa de cura é $\underline{b}$, a constante de taxa de morte causada pela doença é $\underline{c}$, a constante de taxa de morte devido a outras causas é e, e q é a constante que representa a influência da população $R$ na disseminação da infecção. Esses cinco parâmetros são números positivos.

Como $d S(t) / d t+d I(t) / d t+d R(t) / d t=0$, então $S(t)+I(t)+R(t)=N=$ constante, sendo $N$ o número total de indivíduos da população hospedeira. Ou seja, nesse modelo, supõe-se que mortes são imediatamente compensadas por nascimentos. Portanto, o modelo corresponde a um sistema de segunda ordem, visto que $\mathrm{R}(\mathrm{t})=\mathrm{N}-(\mathrm{S}(\mathrm{t})+\mathrm{I}(\mathrm{t}))$.

A inspiração para a forma do termo contendo o parâmetro q veio do trabalho de Turner Jr e Monteiro [8]. O caso $q=0$ já foi analisado por Schimit e Monteiro [7]. Aqui, analisa-se o caso $\mathrm{q}>0$. 


\section{Resultados analíticos}

Os pontos de equilíbrio [3,5], isto é, as soluções estacionárias $(\mathrm{S}(\mathrm{t}), \mathrm{I}(\mathrm{t}), \mathrm{R}(\mathrm{t}))=\left(\mathrm{S}^{*}, \mathrm{I}^{*}, \mathrm{R}^{*}\right)$ do sistema (1)-(3) (com $S^{*}, I^{*}$ e $R^{*}$ constantes) são obtidas a partir de $d S(t) / d t=0$ e $d I(t) / d t=0$ (lembre-se de que $\mathrm{R}(\mathrm{t})=\mathrm{N}-(\mathrm{S}(\mathrm{t})+\mathrm{I}(\mathrm{t}))$ ). Da equação (2), percebe-se que $\mathrm{I}^{*}=0$ torna $\mathrm{dI}(\mathrm{t}) / \mathrm{dt}=0$; consequentemente, da equação (3) tem-se $\mathrm{R}^{*}=0$ e, então, $\mathrm{S}^{*}=\mathrm{N}$. Essa solução estacionária em que $\mathrm{I}^{*}=0$ é chamada de solução livre de doença (já que não há doentes). Solução estacionária com I* $>0$ é chamada de solução endêmica (já que a doença persiste na população). Em termos de $I^{*}$, as soluções endêmicas são as raízes do seguinte polinômio:

$$
\mathrm{A}\left(\mathrm{I}^{*}\right)^{2}+\mathrm{BI}^{*}+\mathrm{C}=0
$$

sendo $A=q b(b+e) / e^{2}, B=1+[b(1-q N) / e], C=N\left(1-R_{0}\right) / R_{0}$ com $R_{0}=a N /(b+c)$. Somente raízes reais e positivas desse polinômio possuem significado biológico. $\mathrm{O}$ valor de $\mathrm{S}^{*}$ correspondente a I* é obtido de:

$$
\mathrm{S}^{*}=\mathrm{N}-\left[(\mathrm{b}+\mathrm{e}) \mathrm{I}^{*} / \mathrm{e}\right]
$$

Obviamente, $\mathrm{R}^{*}=\mathrm{N}-\left(\mathrm{S}^{*}+\mathrm{I}^{*}\right)$. As duas raízes da Equação (4) são:

$$
\begin{aligned}
\mathrm{I}_{1}{ }^{*} & =(-\mathrm{B}+\rho) /(2 \mathrm{~A}) \\
\mathrm{I}_{2}{ }^{*} & =(-\mathrm{B}-\rho) /(2 \mathrm{~A})
\end{aligned}
$$

$\operatorname{com} \rho=\sqrt{B^{2}-4 A C}$. Note que $A$ é positivo, enquanto $B$ e $C$ podem ser positivos ou negativos. Se $\mathrm{C}<0$, a única raiz positiva é $\mathrm{I}_{1}{ }^{*}$, independentemente do sinal de $\mathrm{B}$. Se $\mathrm{C}>0, \mathrm{I}_{1}{ }^{*}$ e $\mathrm{I}_{2}{ }^{*}$ são reais e positivas apenas se $\mathrm{B}<0$ e $\mathrm{B}^{2}>4 \mathrm{AC}$.

A estabilidade local de um ponto de equilíbrio $\left(\mathrm{S}^{*}, \mathrm{I}^{*}\right)$ pode ser determinada a partir dos autovalores da matriz jacobiana J, que é obtida linearizando o sistema em torno de tal solução [3,5]; ou seja:

$$
\mathrm{J}=\left(\begin{array}{ll}
\partial \mathrm{f}_{1}(\mathrm{~S}, \mathrm{I}) / \partial \mathrm{S} & \partial \mathrm{f}_{1}(\mathrm{~S}, \mathrm{I}) / \partial \mathrm{I} \\
\partial \mathrm{f}_{2}(\mathrm{~S}, \mathrm{I}) / \partial \mathrm{S} & \partial \mathrm{f}_{2}(\mathrm{~S}, \mathrm{I}) / \partial \mathrm{I}
\end{array}\right)(\mathrm{S}, \mathrm{I})=\left(\mathrm{S}^{*}, \mathrm{I}^{*}\right)
$$

Segundo o teorema de Hartman-Grobman [3,5], o ponto de equilíbrio $\left(\mathrm{S}^{*}, \mathrm{I}^{*}\right)$ é localmente assintoticamente estável se os dois autovalores $\lambda$ de J têm parte real negativa. Esses autovalores são obtidos a partir $\operatorname{de} \operatorname{det}(\mathbf{J}-\lambda \mathbf{I})=0$, em que I é a matriz identidade. Para um sistema de segunda ordem (em que J e I são matrizes $2 \times 2$ ), mostra-se que tais autovalores são as raízes de $\lambda^{2}-\mathrm{T} \lambda+\Delta=0$ sendo $\mathrm{T}$ o traço e $\Delta$ o determinante da matriz J [5]. Esse polinômio tem raízes com parte real negativa (ou seja, o ponto de equilíbrio correspondente é assintoticamente estável) somente se $\mathrm{T}<0$ e $\Delta>0$ [5].

Para $I^{*}=0$, então $\mathrm{T}=\left(\mathrm{R}_{0}-1\right) /(\mathrm{b}+\mathrm{c})-\mathrm{e}<0, \Delta=\mathrm{e}\left(1-\mathrm{R}_{0}\right) /(\mathrm{b}+\mathrm{c})>0$ se $\mathrm{R}_{0}<1$. Logo, a solução livre de doença é localmente assintoticamente estável se $\mathrm{R}_{0}<1$ e instável se $\mathrm{R}_{0}>1$.

Para a solução endêmica $\mathrm{I}_{1}{ }^{*}$, então $\mathrm{T}=-\mathrm{I}_{1}{ }^{*}(\mathrm{~b}+\mathrm{c}) / \mathrm{S}_{1}{ }^{*}-\mathrm{e}<0, \Delta=\operatorname{ae\rho l}_{1}{ }^{*}>0$. Para $\mathrm{I}_{2}{ }^{*}$, mostra-se que $\mathrm{T}=-\mathrm{I}_{2}{ }^{*}(\mathrm{~b}+\mathrm{c}) / \mathrm{S}_{2}{ }^{*}-\mathrm{e}<0, \Delta=-\mathrm{ae} \rho \mathrm{I}_{2}{ }^{*}<0$. Portanto, $\left(\mathrm{S}_{1}{ }^{*}, \mathrm{I}_{1}{ }^{*}\right)$ é localmente 
assintoticamente estável e $\left(\mathrm{S}_{2}{ }^{*}, \mathrm{I}_{2}{ }^{*}\right)$ é instável (pois $\Delta<0$ ). Note que, no caso em que $\mathrm{R}_{0}<1$ (ou seja, $\mathrm{C}>0$ ), podem existir duas soluções estacionárias localmente assintoticamente estáveis. Entretanto, para isso, é necessário que $\mathrm{B}<0$ e $\mathrm{B}^{2}>4 \mathrm{AC}$.

Observação: não se pode tornar nulo o traço $\mathrm{T}$ associado a $\mathrm{I}_{1}{ }^{*}$ ou $\mathrm{I}_{2}{ }^{*}$, o que é uma condição necessária para a ocorrência de bifurcação de Hopf [3,5]. Portanto, não pode haver ciclo-limite; ou seja, não pode haver oscilação autossustentada nesse sistema.

Em resumo, converge-se para $\left(\mathrm{S}_{1}{ }^{*}, \mathrm{I}_{1}{ }^{*}\right)$ se $\mathrm{R}_{0}>1$. Para $\mathrm{R}_{0}<1$, dependendo da condição inicial, converge-se ou para $(\mathrm{N}, 0)$ ou para $\left(\mathrm{S}_{1}{ }^{*}, \mathrm{I}_{1}{ }^{*}\right)$ se $\mathrm{B}<0$ e $\mathrm{B}^{2}>4 \mathrm{AC}$. Assim, para $\mathrm{R}_{0}<1$, pode haver a convivência de duas soluções estacionárias atratoras.

\section{Simulações numéricas}

A fim de ilustrar os resultados analíticos apresentados na seção anterior, nesta seção são exibidas seis figuras obtidas por simulação numérica. Essas figuras mostram a evolução temporal da quantidade de indivíduos nas classes $S$ (em verde), $I$ (em vermelho) e $R$ (em azul). Aqui, considera-se $\mathrm{N}=1$; ou seja, o tamanho total da população está normalizado, de modo que $S(t), I(t)$ e $R(t)$ representam as porcentagens de suscetíveis, infectados e recuperados no instante $t$.

Essas figuras foram obtidas resolvendo numericamente o sistema (1)-(3) usando o método de integração de Runge-Kutta [5] de quarta ordem, com passo de integração igual a 0,01 . Nessas figuras, $b=1 / 2, c=3 / 10$ e e $=1 / 10$. O objetivo é investigar o efeito da variação dos valores dos parâmetros a e q que são, respectivamente, a constante de taxa de contágio e a constante que reflete a influência dos recuperados. Esses parâmetros, relacionados aos contatos sociais que ocorrem na população, são aqueles cujos valores podem ser mais facilmente controlados por meio de medidas que restrinjam a movimentação da população. Os valores dos parâmetros $\underline{b}, \underline{c}$ e $\underline{\mathrm{e}}$, relacionados a cura e morte, são mais difíceis de serem modificados na prática, pois exigem investimentos em pesquisas por novos medicamentos, investimentos na melhora do sistema de saúde pública, etc.

Na Figura 1(a), a=2/10 e q=1/10; na Figura 1(b), a=2 e q=1/10; na figura 1(c), a=2 e q=1. Assim, de 1(a) para 1(b), o valor de a aumentou; e de 1(b) para 1(c), o valor de $q$ aumentou. Nessas três figuras, a condição inicial é $S(0)=9 / 10, I(0)=1 / 10$ e $R(0)=0$ (assim, inicialmente, a população é formada por $90 \%$ de suscetíveis e $10 \%$ de infectados).

$\mathrm{Na}$ Figura $1(\mathrm{a})$, tem-se $\mathrm{R}_{0}=0,25<1$ e a doença tende a desaparecer naturalmente na população, já que se converge para o ponto de equilíbrio livre de doença. Na Figura 1(b), tem-se $R_{0}=2,5>1$. Nesse caso, a solução atratora obtida numericamente corresponde ao ponto de equilíbrio endêmico dado por $\left(\mathrm{S}_{1}{ }^{*}, \mathrm{I}_{1}{ }^{*}\right)=(0,38 ; 0,10)$, o que está de acordo com os valores calculados a partir das Equações (5) e (6). Assim, aumentando-se a taxa de encontro entre $S$ e $I$ (aumentando o valor de a $)$, a doença pode se tornar endêmica.

Na Figura $1(\mathrm{c})$, tem-se também $\mathrm{R}_{0}=2,5$ e a solução atratora é $\left(\mathrm{S}_{1}{ }^{*}, \mathrm{I}_{1}{ }^{*}\right)=(0,25 ; 0,13)$. Comparando 1(b) com 1(c), observa-se uma diminuição na porcentagem de suscetíveis e um aumento na porcentagem de infectados em regime permanente, graças a um aumento na participação dos recuperados (um aumento no valor de q) na disseminação do agente infeccioso. 
Na figura 2(a), $a=2 / 10, q=16, S(0)=53 / 100, I(0)=7 / 100$ e $R(0)=40 / 100 . N a$ figura 2(b), $a=2 / 10, q=20, S(0)=53 / 100, I(0)=7 / 100$ e $R(0)=40 / 100$. Na figura 2(c), $\mathrm{a}=2 / 10, \mathrm{q}=20, \mathrm{~S}(0)=9 / 10, \mathrm{I}(0)=1 / 10$ e $\mathrm{R}(0)=0$. Nessas três figuras, $\mathrm{R}_{0}=0,25<1$. Comparando 2(a) com 2(b), percebe-se que um aumento no valor de q acima de um número crítico $\mathrm{q}_{\mathrm{c}}$ é suficiente para tornar endêmica a doença, mesmo com $\mathrm{R}_{0}<1$. Nesse caso, o valor crítico de q é 16,7 (obtido de $\mathrm{B}<0$ e $\mathrm{B}^{2}>4 \mathrm{AC}$ ). Comparando 2(b) com 2(c), nota-se que, partindo de outra condição inicial, pode-se convergir para a solução livre de doença mesmo para $\mathrm{q}>\mathrm{q}_{\mathrm{c}}$.
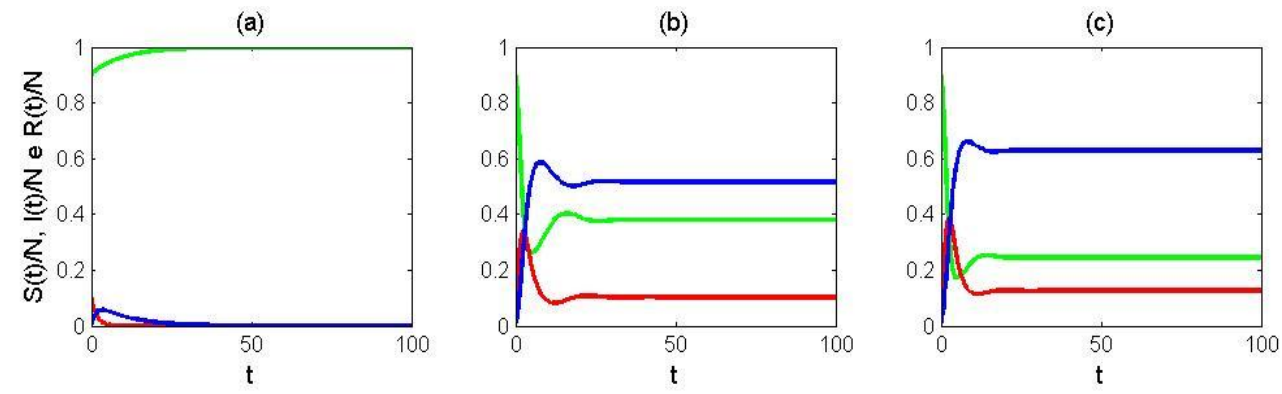

Figura 1: Evoluções temporais de $\mathrm{S}(\mathrm{t}) / \mathrm{N}$ (verde), $\mathrm{I}(\mathrm{t}) / \mathrm{N}$ (vermelho) e $\mathrm{R}(\mathrm{t}) / \mathrm{N}$ (azul) para $\mathrm{N}=1$. Assim, o eixo vertical representa as porcentagens de $S, I$ e $R$. A condição inicial é $(\mathrm{S}(0), \mathrm{I}(0), \mathrm{R}(0))=(9 / 10,1 / 10,0)$. Os valores dos parâmetros são $\mathrm{b}=1 / 2, \mathrm{c}=3 / 10, \mathrm{e}=1 / 10$ e em (a) $a=2 / 10$ e q=1/10; em (b) $a=2$ e q=1/10; e em (c) $a=2$ e q=1.
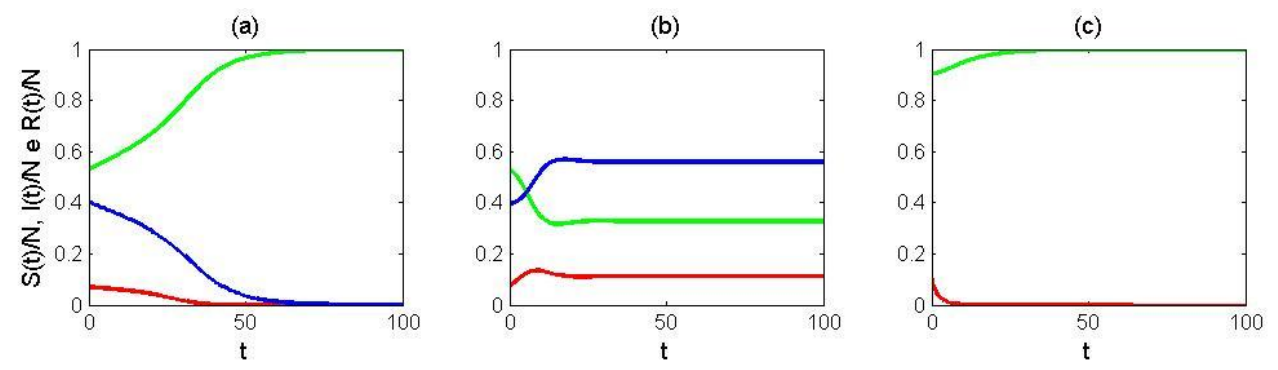

Figura 2: Evoluções temporais de $\mathrm{S}(\mathrm{t}) / \mathrm{N}$ (verde), $\mathrm{I}(\mathrm{t}) / \mathrm{N}$ (vermelho) e $\mathrm{R}(\mathrm{t}) / \mathrm{N}$ (azul) para $\mathrm{N}=1$. Os valores dos parâmetros são $\mathrm{a}=2 / 10, \mathrm{~b}=1 / 2, \mathrm{c}=3 / 10, \mathrm{e}=1 / 10$ e em (a) $\mathrm{q}=16$, $\mathrm{S}(0)=53 / 100, \mathrm{I}(0)=7 / 100$ e $\mathrm{R}(0)=40 / 100$; em (b) $\mathrm{q}=20, \mathrm{~S}(0)=53 / 100, \mathrm{I}(0)=7 / 100 \mathrm{e}$ $\mathrm{R}(0)=40 / 100$; e em (c) $\mathrm{q}=20, \mathrm{~S}(0)=9 / 10, \mathrm{I}(0)=1 / 10$ e $\mathrm{R}(0)=0$.

\section{Conclusões}

Neste trabalho, considerou-se a influência da parcela da população recuperada (e, consequentemente, imune) na disseminação de um agente infeccioso que se propaga via contatos sociais entre suscetíveis e infectados. Talvez, essa influência seja crucial para 
explicar a persistência na população humana de algumas doenças contagiosas típicas de crianças. Aqui, foram obtidos resultados analíticos a respeito das coordenadas e das estabilidades locais dos pontos de equilíbrio, que são as únicas soluções atratoras do modelo proposto. Mostrou-se que, para $\mathrm{R}_{0}>1$, converge-se para o ponto de equilíbrio endêmico $\left(\mathrm{S}_{1}{ }^{*}, \mathrm{I}_{1}{ }^{*}\right)$; para $\mathrm{R}_{0}<1$, converge-se ou para $\left(\mathrm{S}_{1}{ }^{*}, \mathrm{I}_{1}{ }^{*}\right)$ ou para o ponto de equilíbrio livre de doença $(\mathrm{N}, 0)$, dependendo da condição inicial e dos valores dos parâmetros.

Resta verificar se o modelo é, de fato, válido para descrever a dinâmica de doenças contagiosas típicas de crianças. A validade desse modelo pode ser testada confrontando suas previsões com dados epidemiológicos reais, como feito em [8].

\section{Agradecimentos}

Ana L. S. Moraes agradece à CAPES pela bolsa de mestrado. Luiz H. A. Monteiro agradece ao CNPq pela bolsa de produtividade.

\section{Referências}

[1] R. M. Anderson and R. M. May, Infectious Diseases of Humans, Oxford University Press (1991).

[2] K. Dietz and J. A. P. Heesterbeek, Daniel Bernoulli's epidemiological model revisited, Math. Biosci., vol. 180, 1-21 (2002).

[3] J. Guckenheimer and P. Holmes, Nonlinear Oscillations, Dynamical Systems, and Bifurcations of Vector Fields, Springer (2002).

[4] W. O. Kermack and A. G. McKendrick, Contribution to the mathematical theory of epidemics, Proc. Roy. Soc. Lond. A, vol. 115, 700-721 (1927).

[5] L. H. A. Monteiro, Sistemas Dinâmicos, Livraria da Física (2011).

[6] J. D. Murray, Mathematical Biology I: Introduction, Springer (2002).

[7] P. H. T. Schimit and L. H. A. Monteiro, On the basic reproduction number and the topological properties of the contact network: an epidemiological study in mainly locally connected cellular automata, Ecol. Model., vol. 220, 1034-1042 (2009).

[8] P. P. Turnes Jr. and L. H. A. Monteiro, An epidemic model to evaluate the homogeneous mixing assumption, Commun. Nonlinear Sci. Numer. Simulat., vol. 19, 4042-4047 (2014). 